\title{
BMJ Open Possibilities for interprofessional learning at a Swedish acute healthcare ward not dedicated to interprofessional education: an ethnographic study
}

\author{
Ann Hägg-Martinell, ${ }^{\oplus 1}$ Håkan Hult, ${ }^{2}$ Peter Henriksson, ${ }^{3}$ Anna Kiessling ${ }^{3}$
}

To cite: Hägg-Martinell $A$, Hult $\mathrm{H}$, Henriksson $\mathrm{P}$, et al. Possibilities for interprofessional learning at a Swedish acute healthcare ward not dedicated to interprofessional education: an ethnographic study. BMJ Open 2019;9:e027590. doi:10.1136/ bmjopen-2018-027590

- Prepublication history for this paper is available online To view these files, please visit the journal online (http://dx.doi. org/10.1136/bmjopen-2018027590).

Received 30 0ctober 2018 Revised 28 June 2019 Accepted 09 July 2019
Check for updates

(C) Author(s) (or their employer(s)) 2019. Re-use permitted under CC BY-NC. No commercial re-use. See rights and permissions. Published by BMJ.

${ }^{1}$ Department of Health Scinces, Roda Korsets Hogskola, Huddinge, Sweden

${ }^{2}$ Department of Clinical Science, Intervention and Technology, Karolinska Institutet, Stockholm, Sweden

${ }^{3}$ Department of Clinical Sciences, Danderyds Hospital, Karolinska Institutet, Stockholm, Sweden

Correspondence to Dr Ann Hägg-Martinell; ann.hagg.martinell@rkh.se

\section{ABSTRACT}

Objectives Almost all healthcare today is team-based in collaboration over professional borders, and numerous students have work-based learning in such contexts. However, interprofessional learning (IPL) in clinical settings has mostly been systematically explored in specially designed contexts dedicated to interprofessional education (IPE). This study aimed to explore the possibilities for IPL activities, and if or how they occur, in an acute ward context not dedicated to IPE.

Design and setting Between 2011 and 2013 ethnographic observations were performed of medical and nursing students' interactions and IPL during early clerkship at an acute internal medicine ward in Sweden. Field notes were taken and analysed based on the framework of IPE: learning with, from and about. Participants 21 medical, 4 nursing students and 30 supervisors participated.

Results Learning with - there were no organised IPE activities. Instead, medical and nursing students learnt in parallel. However, students interacted with staff members from other professions. Learning from-interprofessional supervision was frequent. Interprofessional supervision of nursing students by doctors focused on theoretical questions and answers, while interprofessional supervision of medical students by nurses focused on the performance of technical skills. Learning about-students were observed to actively observe interactions between staff and learnt how staff conducted different tasks.

Conclusion This study shows that there were plenty of possibilities for IPL activities, but the potential was not fully utilised or facilitated. Serendipitous IPL activities differed between observed medical and nursing students. Although interprofessional supervision was fairly frequent, students were not learning with, from or about each other over professional borders.

\section{BACKGROUND}

Learning takes place in a social context and interactions and interpersonal communications allow an individual to integrate the experience of others into his or her learning. ${ }^{1}$ Work-based learning in hospital settings has the potential to contribute to a socialisation process of medical and nursing students into their future profession. ${ }^{2}$ Healthcare

\section{Strengths and limitations of this study}

- The ethnographic design provided a potential to gain a deeper understanding of participants' actions and interactions, and the extended observation period of large numbers of staff and students increased the findings' trustworthiness.

- Investigator triangulation was applied, and all researchers were engaged in the analysis, which meant that different professional perspectives and experiences could be applied in order to ensure credibility.

- The quality of ethnographic data depends on whether participants feel comfortable to act as if they had not been observed. Therefore, we applied the 'marginal participant" technique, in which the observer plays just a minimal role in the interaction.

- A limitation of the ethnographic design is the risk of researcher bias, as the researcher is used as a tool during the observations.

education should include opportunities to develop skills, behaviours and attitudes needed in interprofessional teamwork. ${ }^{3}$ It is also important to support the development of relationships with other professions during the learning process. ${ }^{4}$

Interprofessional education (IPE) occurs when two or more professions learn with, from and about each other in order to improve collaboration and the quality of practice. ${ }^{56}$ IPE should therefore be an integral part of students' practical experience. ${ }^{7}$

Learning about other professional roles can be acquired when students from different professions are encouraged to interact with each other. ${ }^{7}$ According to Greenstock et $a l^{8}{ }^{8}$ students' first interaction with other health professionals often occurs during clinical placements. In addition, active patient-centred learning achieved by working together taking care of patients in a real ward context seems to be an effective way to increase collaborative and professional competence. ${ }^{9-11}$ 
However, workplace learning for medical and nursing students takes place in an environment primarily organised to effectively take care of patients. ${ }^{12}$ It has been questioned whether interprofessional student-led care is safe for patients. However, a recent study shows that supervised IPE in a patient-based setting can be performed in a level II trauma hospital with satisfactory patient safety. ${ }^{13}$ Supporting factors for learning during interprofessional learning (IPL) in clinical settings are, for example, supervisors experienced in IPE, meeting new people and preparation before the learning activities. ${ }^{14}$ An effective team communication, positive attitude and mutual support of students' IPL are also important. ${ }^{15}$ In addition, to participate in interprofessional collaboration, the possibility to have time to socialise and experience interprofessional collaboration are supportive. ${ }^{16} 17$ Barriers for participation in IPE are, for example, a negative team culture, competitiveness and a negative attitude to IPE. ${ }^{14}$ Furthermore, authoritarian attitudes and hierarchies could be negative factors. ${ }^{18}$

IPE in clinical settings often takes place in contexts structured and dedicated to facilitate interprofessional interaction, and learning and these contexts have been systematically explored. ${ }^{919-21}$ Ericson $e t a l^{22}$ stated that students in general have positive experiences of IPE. Opportunities to learn over professional borders are often appreciated by both medical and nursing students. ${ }^{23} 24$ Hallin and Kiessling ${ }^{21}$ elucidated that a well-organised interprofessional training ward provides a supportive and permissive learning environment where students have opportunities to interact and learn with each other.

In this paper, we defined IPE as organised and scheduled IPL activities based on structured and dedicated IPE concepts. However, we argue that interprofessional competence may be gained within a wider framework. IPL is an educational process through which students and staff are interacting in teamwork over professional borders which offer opportunities for cooperative learning. The aim of such learning is to enable students to obtain knowledge, skills and professional attitudes that they would not be able to obtain effectively in any other way. ${ }^{25}$ IPL includes all forms of learning over professional borders and does not necessarily need to be organised and planned in advance. However, IPL involving undergraduate students in a regular healthcare context, not dedicated to IPE, has to the best of our knowledge not been studied scientifically. Furthermore, most studies on IPE trying to capture learning have used questionnaires or interviews for data collection. The ethnographic method is a combination of empirical investigation and an understanding of social organisation and culture. Through observing, listening and asking questions, the researcher can acquire a good sense of the social structure and processes in an observed setting and start to understand its culture. ${ }^{26}$ Our hypothesis was that a regular healthcare context, not dedicated to IPE, could provide a great potential for students to achieve interprofessional competence. Therefore, the aim of this study was to explore the possibilities for IPL activities, and whether and how such activities occurred in an acute medical ward not dedicated to IPE.

\section{METHOD}

\section{Study design}

An ethnographic study design was chosen through direct observation of behaviour. This is a powerful but underutilised method to explore interpersonal interactions during workplace learning. The 'marginal participant' technique was applied, in which the observer plays only a minimal role in the social activities that they are observing. ${ }^{26}$

A wide data collection was performed during 20112013 through observing workplace culture in an acute internal medical ward, including students' interactions and opportunities to learn within this context. Inspired by Hammersley and Atkinson, ${ }^{26}$ repeated observations were performed by participating in the daily life of the ward for an extended period of time, to watch what happened, to listen to what was said and to ask questions intended to throw light on whether IPL concerning medical and nursing students occurred at the ward. Focus for the analysis in the present study was to explore actual and possible IPL activities in this context. Based on the same wide data collection, we have previously described the general organisation, learning environment and culture of this ward and medical students' opportunities to participate and learn in this context. ${ }^{27} 28$

\section{Study context}

This study was performed on an acute medical ward in a teaching hospital in Sweden. During the studied period, the assignment of the hospital was to perform acute healthcare, for a population of $\sim 500000$ inhabitants and to perform clinical research and education. The observed ward belonged to the Department of Internal Medicine, one of the largest departments of the hospital. The focus of the ward was the diagnosis and treatment of patients with internal medicine diseases, mainly severe infections, obstructive pulmonary disease, deep venous thrombosis including pulmonary embolism, treated for overdoses, convulsive disorders and allergic reactions. The average treatment period was $<24$ hours. An interprofessional team including a specialist doctor, a junior doctor, a nurse and an auxiliary nurse took care of the patients. They performed paperwork and planning at an office with windows that allowed observation of the patients. Table 1 shows an overview of a working day in the ward.

Permission to perform the ethnographic study on this ward was given by the head of department. Informed consent was obtained from all students, staff, patients and relatives in accordance with the Declaration of Helsinki after information about the purpose, method and that participation was voluntary. The students received oral information from their main supervisor and from the first author; specialist doctors and junior doctors received oral information from the first author. All students who performed work-based learning on the ward during the 


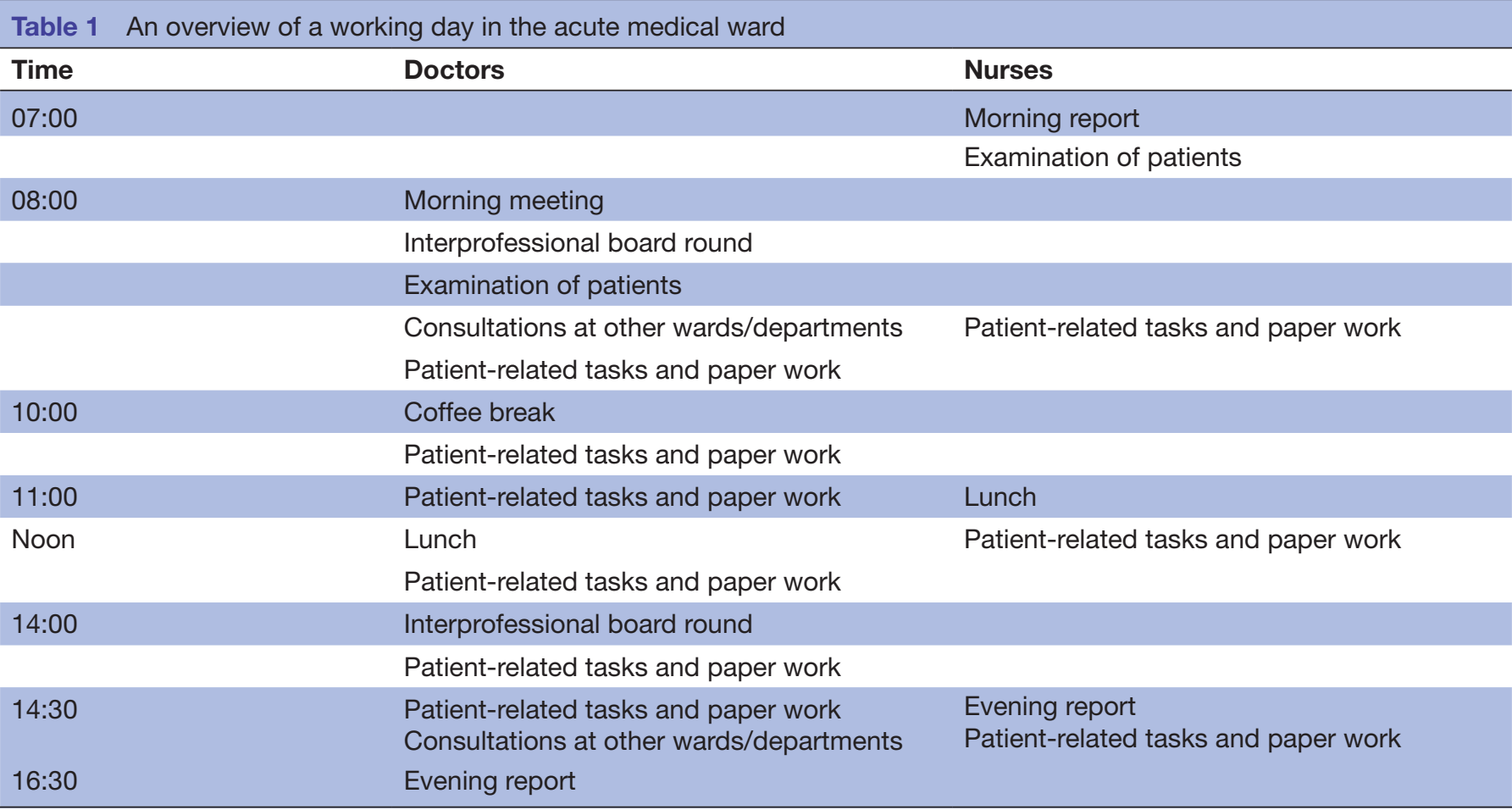

study period agreed to participate. Also, all supervisors and staff agreed to participate. All students, supervisors and staff were informed that the observer was a registered nurse, but that her role at the ward was to conduct research and not to act as a nurse or as a supervisor. All patients and their relatives received information that education and research were performed at the hospital. More specific information about this study was given by the specialist doctor in charge or by the first author.

\section{Data collection}

In total, 27 observations were conducted from early autumn 2011 to early spring 2013. As the observer was a part time researcher, a consecutive sampling of available observation days was applied, based on if students were present and if the observer had scheduled time for research work. Observations were performed only when students were present, that is, during morning, afternoon and evening shifts, Monday to Friday.

Each observation session lasted on average 3-4 hours. The total observation time was about 100 hours and included 50-hour interprofessional board rounds, 25-hour collaborative paperwork in offices, 20-hour patient care and consultations and at least 5-hour informal patient-related interactions, for example, in the corridor, in the staff lounge, in the medical room or in the treatment room. No formal interviews were conducted. However, when needed, short informal questionings were conducted with participants, aiming to clarify thoughts, to ask for explanations and to get a deeper understanding of the observations.

In total, 21 medical students, 4 nursing students and 30 supervisors (specialist doctors, junior doctors and nurses) were observed. Both male and female students participated. Medical students were in term 5 and 6 (out of 11) and were placed on the ward for 1-5 days. Nursing students were in term six (out of six) and were placed on the ward for 4-8 weeks. The difference in length of stay explains the difference in number of observed medical and nursing students. On the other hand, the four nursing students were recurrently observed on many occasions. In addition, auxiliary nurses, patients and relatives could be observed when interacting with students. Individual students could be observed on several occasions. The last observations mostly yielded repetitive information, thus saturation was assumed. Field notes were taken during the observations, based on a structured observation protocol. The content focused on the aim of the study. As the span of memory for field observations is short, it is important that the notes are written as soon after the event as possible. ${ }^{29}$ Therefore, the notes were transcribed later the same day. ${ }^{26}$ The first author performed all observations and informal questionings wearing hospital clothes. She had no relationship to the participants as a nurse or a supervisor either before, during or after the observations. Her only relationship to the participants was as a researcher.

\section{Data analysis}

An inductive approach was used with the purpose to inform the focus of later observations. A preliminary data analysis started early during the fieldwork period based on observations made when both medical and nursing students were present at the ward. When the analysis revealed findings that needed to be studied in more detail, the data collection was modified, for example, by 
adding observations of nursing students interacting with auxiliary nurses. The final analytical process involved all field notes. A deductive content analysis was conducted beginning with an expected pattern based on the framework of IPE: learn with, from and about others. ${ }^{5}$ This framework was then tested against the observations in a back and forth process. Broad transcriptions enabled assembly of topic-focused data sets. The analysis of the data involved four steps: familiarisation with the data by reading transcripts; developing codes; sorting of codes into categories based on similarities of content and meaning and finally, grouping of categories into themes based on the IPE framework. The original framework aims to describe peer-learning over professional borders. However, all observations, including possibilities for, or actual interprofessional interactions, were included in the analysis.

The first author was aware of, and in the analysis strove to allow for, a potential influence on the participants behaviour. The large amount of observation data combined with the opportunity to move back and forth between the transcriptions in the emerging analysis formed a good base for understanding of learning situations and interactions. The first author coded all data according to the framework. The findings were then repeatedly discussed in the research group in a reflective process, where their different perspectives and experiences contributed to the interpretation of the data. The process continued until agreement was reached. To improve the appraisal of transferability, the setting, data collection and analysis process were carefully described. ${ }^{30}$ Examples of field notes were presented to reinforce the analytical findings. Certain linguistic and grammatical revisions were also carried out when the field notes were translated into English. ${ }^{31}$

\section{Patient and public involvement}

There was no patient or public involvement in the conduct of this study. Patients and the public were not involved in the design or planning of the study.

\section{RESULTS}

\section{Learn with}

This theme illustrates medical and nursing students' opportunities to learn and interact with students and staff of other professions at the ward.

The circumstances of students' placements at the ward influenced whether and how they became involved in different activities. Even if both medical and nursing students were in the same ward at the same time, their prerequisites for interaction with others differed. They had, for example, different lengths of placements, lengthiest for nursing students and they had different supervision models. The medical students followed selected patients and were supervised by the doctor in charge, while the nursing students had two designated supervisors to follow.
Medical and nursing students were observed learning in parallel without direct interaction with each other. In situations when students became involved in the direct patient care, they collaborated and learnt together with their supervisors, but not with students of other professions. For example, when the nursing and the medical students participated in the same ward round, they did not interact with each other and the only structured interprofessional interactions observed were with staff members, such as when a medical student interacted with a nurse. The only situations where medical and nursing students interacted with each other were during informal patient-related indirect discussions, for example, in the staff lounge.

There were several possibilities such as board rounds, collaborative paperwork and patient care situations, when interprofessional peer to peer learning could have been supported.

On several occasions, consultants from other departments arrived to examine patients on the ward. On one occasion, an orthopaedic surgeon arrived to examine infected wounds, and both a medical and a nursing student observed the examination.

A patient was treated for an overdose and had several infected wounds. An specialist doctor from the orthopaedic department arrived to examine the wounds. $\mathrm{He}$, the junior doctor at the ward, and the medical student, put on aprons. The two doctors leaned over the patient and palpated and squeezed the wounds. The medical and nursing students were standing on oppositesides of the room. The medical student was not invited to participate in the examination and just stood quietly in her apron beside them. The nursing student did not participate either. She just stood at her side of the room and observed, not wearing an apron. Observation note (a specialist doctor, a junior doctor a medical student, a nurse and a nursing student).

The lack of physical space prevented medical and nursing students' interaction and collaboration with each other. There was a lack of computers and desks for both students and staff. The students often had to sit behind their supervisors during board rounds. It was only when some of the staff left the office that the students got the opportunity to use a computer or a desk. There were no silent rooms for small-group discussions or reflections. However, there was a peaceful atmosphere which was facilitated by several circumstances. The teamwork processes were smoothly organised with scheduled ward rounds, fixed locations for different activities and so on. Checklists and other tools simplified patient care. In this interprofessional shared platform for collaboration and patient care, it would have been natural to involve students. However, there were several situations with unexploited opportunities for IPL, especially situations when both medical and nursing students participated. 


\section{Learn from}

This theme describes medical and nursing students' opportunities to learn from each other and from staff of other professions.

The students were able to learn from a variety of staff over professional borders. During the clinical placement in the acute medical ward, both medical and nursing students interacted and learnt regularly from external professional experts, for example, specialist doctors, physiotherapists, occupational therapists and counsellors. Both medical and nursing students expressed that interprofessional supervision was important, and mentioned that they received insight into both teamwork and the roles of other professions.

Differences and hierarchies between medical and nursing students were observed. As a consequence, some students were bypassed, and opportunities for learning from others were often missed. Some of the hierarchies impaired the working climate and thereby created barriers to learning. Medical students were observed taking precedence over nursing students. There were several occasions when nursing students had to interrupt their learning activities to give space for medical students to participate. For example, a nursing student was sitting in the observation area, writing up a medical record on the computer. She was expected to interrupt her writing so that a medical student could use the computer. Another situation occurred when a nursing student was about to take a blood test but was asked to take a step backward so that a medical student could take it instead.

Interprofessional supervision was common at the ward and was appreciated by both medical and nursing students. During ward rounds, medical students were sometimes invited to participate in the discussion, for example, by answering questions on medical treatments or results of different investigations, while nursing students often merely watched. However, during other ward rounds, nursing students could be active by taking the nurse's role in the session. This was especially apparent when individual specialist doctors or junior doctors invited them to participate or during more informal afternoon shift handovers. In addition, nursing students were given different opportunities to ask questions in different situations. During some round sessions, the students were asked if they had any further questions. During other round sessions, the students only watched. Based on the observations, it seemed random whether or not students were invited to participate and learn from interprofessional round dialogues.

Interprofessional supervision of nursing students by doctors involved, for example, questions and answers about theory and how to perform medical examinations. The nursing students sometimes got the opportunity to act independently as a nurse during board rounds. For example, a discussion took place between a nursing student and a specialist doctor where the nursing student independently performed a medical board round with the specialist doctor. Another dialogue took place between a nursing student and a junior doctor, and the nurse only made certain clarifications to the junior doctor. Observations indicated that such interprofessional supervision occurred when the nurse supervisor let the student act independently as a nurse by standing back and observing. In addition, the supervising nurse had to trust the students' knowledge and skills to perform nursing tasks in interaction with a doctor. For example, a nursing student was observed performing a board round with a junior doctor.

During the board round the team discusses individual care plans for their patients. The nursing student is active in the dialogue with the junior doctor and the nurse makes only certain contributions in the dialogue. Observation note (a nursing student, a nurse and a junior doctor).

Interprofessional supervision of medical students by nurses focused on how to perform certain technical tasks such as collecting blood samples and performing orthostatic blood pressure assessments. For example, a medical student was observed performing an orthostatic blood pressure assessment on a patient.

A medical student was to perform an orthostatic blood pressure assessment on a patient. The nurse informs the medical student and the patient how the procedure should be performed, what to look for, and what to consider regarding body position (lying, sitting, standing), intervals of time (minute intervals), and changes to be observed (blood pressure, pulse, dizziness). Observation note (a medical student and a nurse).

The nursing students worked closely together with their nurse supervisors and with auxiliary nurses. The cooperation with the auxiliary nurses provided many opportunities for interprofessional supervision. Auxiliary nurses' supervision of nursing students focused on basic patient care. For example, one situation of bed making and how to move a bed-bound patient in the bed was observed.

An auxiliary nurse is introducing a nursing student to how to make up a patient bed, since the bed was very messy. The auxiliary nurse gives practical instructions on how to move the patient in bed, on how to put on new sheets and on how to finish the task. Observation note (an auxiliary nurse and a nursing student).

\section{Learn about}

This theme illustrates medical and nursing students' opportunities to learn about other professions on the ward.

Students were observed actively watching and learning about the staff of other professions and how these professionals performed their clinical skills, interacted with each other and communicated with patients and relatives. When the medical and nursing students arrived at the ward they had to adapt to the ongoing activities. 
Observations indicated that they were often thrown directly into already ongoing situations, without opportunities for supervisors to allocate time for introduction. There was also no time for students to ask questions in these situations.

The nurses started their morning shift and the supervisor informed the nursing student that Monday mornings are quite busy, so the student just has to perform their duties. The nursing student seemed to accept the situation. One hour later, the doctor team arrived and joined the morning meeting where the head nurse went through the day's schedule, the number of patients, welcomed two new medical students to the ward, and divided the patients between the different teams. The specialist doctor and the junior doctor informed the medical student in their team that on Monday mornings there is a heavy workload, so the student had to catch on to the team duties without any further introduction at the moment. The student was not shown or told anything about what was going to happen. The medical student seemed to accept the situation and followed the supervisor to the first patient they should examine. Observation note (the staff at the ward, a medical and a nursing student).

In addition, students were observed watching and learning when the staff at the ward interacted with consultants from other departments who visited the ward. However, no students were observed interacting or asking questions, they were just observers during these consultations. For example, a junior doctor from the emergency department arrived at the ward. The junior doctor reported a new patient to the team.

A junior doctor arrived from the emergency department with a new patient and started handing over a report to the team. It was a woman with convulsive disorder and a previous myocardial infarction. A dialogue took place between the junior doctor from the emergency department, the nurse and the junior doctor at the ward. The medical student was not invited to or involved in the dialogue, but he actively watched and listened and made notes about the patient. Observation note (two junior doctors, a nurse and a medical student).

\section{DISCUSSION}

The studied learning environment provided great opportunities for valuable IPL. However, no such activities were organised or facilitated and merely occurred randomly. Most of the time medical and nursing students were just learning in parallel.

Learning with others aims to create a shared platform for collaboration and communication.

As described in a previous paper, ${ }^{27}$ the staff working at the ward chosen for this study had to handle numerous complex and stressful situations in a smooth teamwork processes. The findings from the present study show that interprofessional collaboration and communication that occur daily can be underused as learning opportunities for medical and nursing students. For example, medical and nursing students could have opportunities to perform medical board rounds together. Furthermore, they could, for example, perform medical examinations on patients together. We argue that acute wards such as the observed one, have a potential to support high quality IPL, but currently this potential has not been fully utilised.

The observed lack of physical space at the studied ward not designed for IPE could also hinder students' participation and learning, and impair their abilities to collaborate with each other. The need of a physical learning space was emphasised by Hallin and Kiessling ${ }^{21}$ who stated that students who had their work-based learning in wards organised and equipped for IPE perceived that they were in a safe place with space for IPL.

Learning from others has a potential to expand one's own competence both laterally and vertically. The participants had opportunities to interact with and learn from other professions working in the ward. Hägg-Martinell et $a l^{2328}$ have pointed out that medical and nursing students valued interprofessional supervision as important during their work-based learning. However, we argue that observed hierarchies and differences between student categories were influenced by the interdisciplinary and interprofessional hierarchies that were observed at the ward. Langendyk $e t a \vec{l}^{2}$ have argued that medical and nurse educators aim to convey strong occupational identities to their students. This may perpetuate some hierarchical disciplinary boundaries. It would facilitate development of professional competence if health profession educators could move from an uniprofessioal perspective and create IPL opportunities for medical and nursing students. Supervisors at the ward could educate both medical and nursing students when they perform their specific tasks. For example, specialist doctors and junior doctors could educate nursing students in how to listen to heart beats. Furthermore, nurses could educate medical and nursing students when they perform blood tests.

The results of this study indicate that students need to be invited and involved in patient care together with others in an interprofessional team and thereby optimise the learning of collaboration in teams. This is supported by Brennan and Enns ${ }^{33}$ who stated that social relationship and communication contribute to a rewarding collaboration.

The observed different lengths of placement between medical and nursing students might hinder their opportunities to build relationships with each other. Furthermore, we could argue that the short placements of the observed medical students prevented them from taking an active role in the interprofessional teamwork. As the nursing students had longer placements at the ward, it was easier for them to take an active role in the 
interprofessional teamwork. A study by Hägg-Martinell $e t$ $a l^{28}$ has shown that medical students envied the situation of nursing students with longer and more coherent placements. On the other hand, Liljedahl et $a l^{2}$ argued that medical students were adapted to the frequent switching of supervisors and placements.

Learning about others means to decentralise from your own perspective to better understand the perspective of others. We argue that to switch between one's active participation in team-work and active observation of staff as role models in interprofessional care has a potential to contribute to understanding of interprofessional care and to increase knowledge about others. This is somewhat contradictory to the findings by Dornan $e t a l^{34}$ who stated that work-based learning could be accomplished at various activity grades, from passive observation to active performance. He argues that being an active participant in different learning activities, such as interacting with patients, has a potential to improve professional practice. But passive learning activities, such as observing or listening, did not seem to have the potential to contribute to improved professional behaviour. ${ }^{35}$ Observed students in our study mixed various levels of activity in their learning, even if most of the IPL was rather passive. However, the potential of extended student active IPL was obvious in the studied context.

The main finding of this study is that the learning environment provided plenty of possibilities for IPE but that this potential was not fully utilised or facilitated. This has to be evaluated through the lens of the work-place culture and the community of practice ${ }^{36}$ in this particular type of ward. We explored the learning culture in this context in a previous paper. ${ }^{27}$ The workplace culture at the ward was stimulating but complex, time-scarce and ever changing. Inspired by Wenger's community of practice theory, ${ }^{36}$ we described the culture as characterised by complex and stressful situations stabilised by routines and carriers of culture. Variable composition and roles of community members, and transitions through community boundaries were a part of the daily routine, and hierarchies and orders of priority were present as regulators of roles, routines and interactions.

\section{Strengths and limitations}

The ethnographic design and the extended observation period of many staff and student interactions increased trustworthiness and credibility of the findings. A limitation of the observational method is the risk of bias of the researcher, as the researcher is used as a tool during the observations. The observer (the first author) was a female registered nurse with long experience of nurse education in acute internal medicine care settings. This preunderstanding of context might allow added depth in both observations and analysis. By contrast, this could be a bias that might mean that important aspects remain unnoticed. Using the marginal participant technique, in which the observer plays only a minimal role in the interaction, would minimise the risk of disturbing what happens in the room, how participants are placed, and which interactions that took place. Furthermore, investigator triangulation applied different professional perspectives and experiences in the analysis that would ensure credibility. It can be seen as a limitation that we performed the analysis based on the established framework of IPE: learning with, from and about. However, we see this paper as a base for future research aiming to a more inductive exploration of IPL in ordinary care contexts. We studied medical and nursing students IPL during undergraduate placement at an acute internal medicine care ward and thus the results might be transferable to undergraduate learning in similar healthcare settings.

\section{CONCLUSION}

Plenty of possibilities for IPL activities were observed in this acute medical ward not dedicated to IPE, but the potential was not fully utilised or facilitated. IPL activities differed between observed medical and nursing students. Although interprofessional supervision was fairly frequent, students were not learning with each other over professional borders.

Acknowledgements The authors thank all the participants for giving their time to take part in this study.

Contributors AK took initiative to the study and $\mathrm{HH}$ came up with the idea to apply an ethnographic design. AHM performed, under supervision of AK, all observations and acquisition of data and compiled the written documentation of the observational data. All four authors (AHM, $\mathrm{HH}, \mathrm{PH}$ and $\mathrm{AK}$ ) contributed substantially to the analysis and interpretation of data. AK and AHM performed the main part of drafting the work and then all authors (AHM, $\mathrm{HH}, \mathrm{PH}$ and $\mathrm{AK}$ ) contributed substantially in revising it critically for important intellectual content. All four authors (AHM, $\mathrm{HH}, \mathrm{PH}$ and $\mathrm{AK}$ ) have approved the final submitted version and all four authors (AHM, HH, PH and AK) have agreed to be accountable for all aspects of the work in ensuring that questions related to the accuracy or integrity of any part of the work are appropriately investigated and resolved.

Funding The study was supported by grants provided by the Stockholm County Council (ALF project).

Competing interests None declared.

Patient consent for publication Not required.

Ethics approval The study was approved by the Regional Ethical Review Board in Stockholm, Sweden (No 2011/1268-31/5).

Provenance and peer review Not commissioned; externally peer reviewed. Data availability statement Data are available upon reasonable request.

Open access This is an open access article distributed in accordance with the Creative Commons Attribution Non Commercial (CC BY-NC 4.0) license, which permits others to distribute, remix, adapt, build upon this work non-commercially, and license their derivative works on different terms, provided the original work is properly cited, appropriate credit is given, any changes made indicated, and the use is non-commercial. See: http://creativecommons.org/licenses/by-nc/4.0/.

\section{REFERENCES}

1. Vygotskij L. The collected works of L.S. Vygotsky. The history of the development of higher mental functions. New York: Plenum Press, 1997.

2. Liljedahl M, Boman LE, Fält CP, et al. What students really learn: contrasting medical and nursing students' experiences of the clinical learning environment. Adv Health Sci Educ Theory Pract 2015;20:765-79.

3. Lumague M, Morgan A, Mak D, et al. Interprofessional education: the student perspective. J Interprof Care 2006;20:246-53. 
4. Bok HGJ, Teunissen PW, Favier RP, et al. Programmatic assessment of competency-based workplace learning: when theory meets practice. BMC Med Educ 2013;13:123.

5. CAIPE. Interprofessional education - a definition, 2002. Available: www.caipe.org

6. WHO. Framework for Action on Interprofessional Education \& Collaborative Practice, 2010. Available: https://apps.who.int/iris/ handle/10665/70185

7. Armitage $H$, Pitt $R$, Jinks A. Initial findings from the TUILIP (Trent universities interprofessional learning in practice) project. $J$ Interprof Care 2009;23:101-3.

8. Greenstock L, Brooks P, Malloy E, et al. Medical students' perceptions of role models on clinical placements. Clin Teach 2014;11:104-8.

9. Hallin K, Kiessling A, Waldner A, et al. Active interprofessional education in a patient based setting increases perceived collaborative and professional competence. Med Teach 2009;31:151-7.

10. Bridges D, Davidson RA, Soule Odegard P, et al. Interprofessional collaboration: three best practice models of interprofessional education. Med Educ Online 2011;16:6035.

11. Bharamgoudar R, Sonsale A. Twelve tips for medical students to make the best use of ward-based learning. Med Teach 2017;39:1119-22.

12. Mennin S. Self-Organisation, integration and curriculum in the complex world of medical education. Med Educ 2010;44:20-30.

13. Hallin $\mathrm{K}$, Gordon $\mathrm{M}$, Sköldenberg $\mathrm{O}$, et al. Readmission and mortality in patients treated by interprofessional student teams at a training ward compared with patients receiving usual care: a retrospective cohort study. BMJ Open 2018;8:e022251.

14. Telford M, Senior E. The experiences of students in interprofessional learning. Br J Nurs 2017;26:350-4.

15. Brock D, Abu-Rish E, Chiu C-R, et al. Interprofessional education in team communication: working together to improve patient safety. BMJ Qual Saf 2013;22:414-23.

16. Reeves S, Freeth D, McCrorie P, et al. 'It teaches you what to expect in future ... ': interprofessional learning on a training ward for medical, nursing, occupational therapy and physiotherapy students. Med Educ 2002;36:337-44.

17. Mitchell M, Groves M, Mitchell C, et al. Innovation in learning - An inter-professional approach to improving communication. Nurse Educ Pract 2010;10:379-84.

18. Friman A, Wiegleb Edström D, Edelbring S. Attitudes and perceptions from nursing and medical students towards the other profession in relation to wound care. J Interprof Care 2017;31:620-7.

19. Falk AL, Hult H, Hammar M, et al. One site fits all? A student ward as a learning practice for interprofessional development. $J$ Interprof Care 2013;27:476-81.
20. Lachmann H, Ponzer S, Johansson U-B, et al. Capturing students' learning experiences and academic emotions at an interprofessional training ward. $J$ Interprof Care 2013;27:137-45.

21. Hallin $K$, Kiessling A. A safe place with space for learning: experiences from an interprofessional training ward. $J$ Interprof Care 2016;30:141-8.

22. Ericson A, Löfgren S, Bolinder G, et al. Interprofessional education in a student-led emergency department: a realist evaluation. $J$ Interprof Care 2017;31:199-206.

23. Hägg-Martinell $A$, Hult $H$, Henriksson $P$, et al. Students perceive healthcare as a valuable learning environment when accepted as a part of the workplace community. Educ Health 2014;27:15-23.

24. Woermann U, Weltsch L, Kunz A, et al. Attitude towards and readiness for interprofessional education in medical and nursing students of Bern. GMS J Med Educ 2016;33.

25. Funnel P. Exploring the value of interprofessional shared learning. In: Soothill K, Mackay L, Webb C, eds. Interprofessional relations in health care. London: Edward Arnold, 1995: 163-71.

26. Hammersley M, Atkinson P. Ethnography: principles in practice. 3rd ed. London: Routledge, 2007.

27. Hägg-Martinell $A$, Hult $H$, Henriksson $P$, et al. Community of practice and student interaction at an acute medical ward: an ethnographic study. Med Teach 2016:38:793-800.

28. Hägg-Martinell A, Hult $\mathrm{H}$, Henriksson $\mathrm{P}$, et al. Medical students' opportunities to participate and learn from activities at an internal medicine ward: an ethnographic study. BMJ Open 2017;7:e013046.

29. Atkinson P. The clinical experience: the construction and reconstruction of medical reality. 2nd ed. Aldershot: Ashgate, 1997.

30. Patton M. Qualitative research \& evaluation methods: integrating theory and practice. London: Sage, 2015.

31. Kvale S, Brinkmann S. Interviews: learning the craft of qualitative research interviewing. Los Angeles: Sage Publications, 2015.

32. Langendyk V, Hegazi I, Cowin L, et al. Imagining alternative professional identities: reconfiguring professional boundaries between nursing students and medical students. Acad Med 2015;90:732-7.

33. Brennan AA, Enns JT. When two heads are better than one: interactive versus independent benefits of collaborative cognition. Psychon Bull Rev 2015;22:1076-82.

34. Dornan $\mathrm{T}$, Boshuizen $\mathrm{H}$, King $\mathrm{N}$, et al. Experience-Based learning: a model linking the processes and outcomes of medical students' workplace learning. Med Educ 2007:41:84-91.

35. Thomson O'Brien MA, Freemantle N, Oxman AD, et al. Continuing education meetings and workshops: effects on professional practice and health care outcomes. Cochrane Database Syst Rev 2001;(2):CD003030.

36. Wenger E. Communities of practice : learning, meaning, and identity. Cambridge: Cambridge University Press, 1998. 